\title{
Fully integrated micro Coriolis mass flow sensor operating at atmospheric pressure
}

\author{
J.C. Lötters ${ }^{1,2}$, T.S.J. Lammerink ${ }^{1}$, J. Haneveld ${ }^{1}$, T.A.G. Hageman ${ }^{1}$ and R.J. Wiegerink ${ }^{1}$ \\ ${ }^{1}$ MESA+ Institute for Nanotechnology, University of Twente, The Netherlands \\ ${ }^{2}$ Bronkhorst HighTech BV, Ruurlo, The Netherlands
}

\begin{abstract}
This paper discusses the design and realization of a micromachined micro Coriolis flow sensor with integrated electrodes for both electrostatic actuation and capacitive readout. The sensor was realized using semicircular channels just beneath the surface of the silicon wafer. The channels have thin silicon nitride walls to minimize the channel mass with respect to the mass of the moving fluid. A comb-shaped electrode design is used to prevent squeezed film damping so that the sensor can operate at atmospheric pressure, thus eliminating the need for vacuum packaging. The new sensor chip no longer requires large external magnets and the size of the chip itself has been reduced to $7.5 \times 7.5 \mathrm{~mm}^{2}$.
\end{abstract}

\section{INTRODUCTION}

Integrated microfluidic systems have gained interest in recent years for many applications including analytical, (bio)chemical, medical, automotive, and industrial devices. A major reason is the need for accurate, reliable, and cost-effective liquid and gas handling systems with increasing complexity and reduced size. In these systems, flow sensors are generally one of the key components.

Most MEMS flow sensors are based on a thermal measurement principle. It has been demonstrated [1-3] that such sensors are capable of measuring liquid flow down to a few $\mathrm{nl} / \mathrm{min}$. These sensors require accurate measurement of very small flow-induced temperature changes. An important problem of thermal flow sensors is that the measurement is highly dependent on temperature and fluid properties like density and specific heat so that the sensor needs to be calibrated for each specific fluid and cannot be used for varying mixtures of fluids. Coriolis type flow sensors, i.e. flow sensors containing a vibrating tube segment in which a mass flow is subject to Coriolis forces, do not have this problem. The Coriolis forces are directly proportional to the mass flow and independent of temperature, pressure, flow profile and fluid properties so that Coriolis flow sensors measure true mass flow.

Coriolis flow meters [4-6] are mostly used for measuring large flow rates, since the relatively weak Coriolis forces are correspondingly harder to detect for small flows. In general, the signal to noise ratio is very sensitive to fabrication and construction errors, as well as external influences of temperature and mechanical nature. In [7] we proposed to fabricate a micro Coriolis mass flow sensor using silicon nitride as the tube material. This resulted in a very thin $(1.2 \mu \mathrm{m})$ tube wall, so that the mass of the tube is small compared to the mass of the moving fluid. This was a significant improvement over [8] and [9], which use silicon as thetube material, leading to a relatively heavy and stiff tube. We demonstrated that a silicon nitride sensor could reach a resolution in the order of 10 milligram/hour [7], however at that time no readout structures were integrated and a Polytec MSA-400 laser vibrometer was needed to optically measure the out-of-plane Coriolis motion of the tube. In [10, 11], we added an integrated capacitive readout, but actuation was done using Lorentz forces in combination with external magnets. In this paper, we show that using an additional pair of electrodes the Lorentz actuation can be replaced by electrostatic actuation, thus eliminating the need for external magnets. The electrodes are comb-shaped, in order to prevent squeezed film damping that would occur using a parallel plate configuration. As a result, the sensor can operate at atmospheric pressure and does not require vacuum packaging.

\section{OPERATING PRINCIPLE}

A Coriolis type flow sensor consists of a vibrating tube. Figure 1 shows schematic drawings of the new Coriolis sensor based on electrostatic actuation and capacitive sensing. The figure illustrates two operation modes of the same mechanical structure. In situation (a), the tube is actuated in a torsion mode indicated by $\omega_{x}$. In (b) it is actuated in a flapping mode indicated by $\omega_{y}$. In both cases, actuation is done by applying suitable voltages $V_{1}$ and $V_{2}$ to capacitors at the inside of the rectangular loop. A mass flow $\Phi_{m}$ inside the tube induces Coriolis forces that excite the other vibration mode, i.e. actuation of the torsion mode results in Coriolis forces that actuate the flapping mode and vice-versa. The Coriolis forces and resulting movements of the tube are proportional to both the actuation amplitude and the mass flow. Both the actuation and the Coriolis movements are detected using capacitors $C_{1}$ and $C_{2}$ at the outside of the loop. The mass flow can be extracted from the two output signals by detecting the phase difference, which is exactly proportional to the amplitude ratio of the Coriolis and actuation movements. 


\section{FABRICATION}

[11].

Here we give a brief summary of the fabrication process. A more detailed description can be found in

Starting with a highly doped $<100>\mathrm{p}++$ wafer, a $500 \mathrm{~nm}$ thick low stress LPCVD silicon-rich silicon nitride (SixNy) layer is deposited. Then the fluidic inlet/outlet holes are etched from the backside of the wafer using the SixNy layer at the top side as etch stop (Fig. 2a). Next, a $1 \mu \mathrm{m}$ thick TEOS (tetraethyl orthosilicate) oxide layer is deposited and removed from the front side of the wafer. Then a $50 \mathrm{~nm}$ layer of chromium is sputtered on the front side of the substrate. This chromium layer is patterned using a mask containing arrays of $5 \times 2 \mu \mathrm{m}$ holes, spaced $3 \mu \mathrm{m}$ apart. This pattern forms the centerline of the final channel. The pattern is then transferred into the nitride layer by reactive ion etching and subsequently the channels are etched in the silicon using isotropic plasma etching (Fig. 2b). The TEOS layer and chromium mask are then removed and another SixNy layer is grown with a thickness of $1.8 \mu \mathrm{m}$ to form the channel walls and seals the etch holes in the first nitride layer (Fig. 2c). A 10/200 nm layer of chromium and gold is sputtered (chromium serving as the adhesion layer for gold) and patterned to create the metal electrodes for actuation and readout (Fig. 2d). Next, the release windows are opened by reactive ion etching of the SixNy layer (Fig. $2 \mathrm{e}$ ) and the structure is released by isotropic silicon plasma etching (Fig. 2f)).

Figure 3 shows a photograph of the fabricated sensor chip. The sensor tube with dimensions $L_{x}=2.5 \mathrm{~mm}$ and $L_{y}=4 \mathrm{~mm}$ is clearly visible in the center of the chip. The tube diameter is approximately $40 \mu \mathrm{m}$. The entire chip measures $7.5 \mathrm{~mm} \times 7.5 \mathrm{~mm}$.

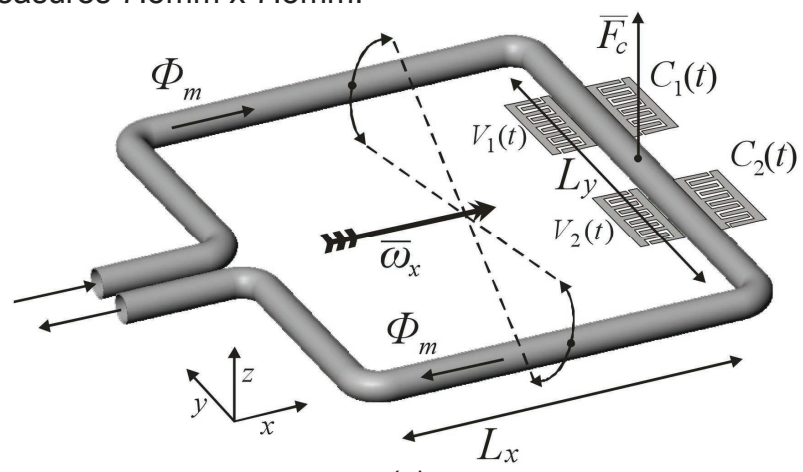

(a)

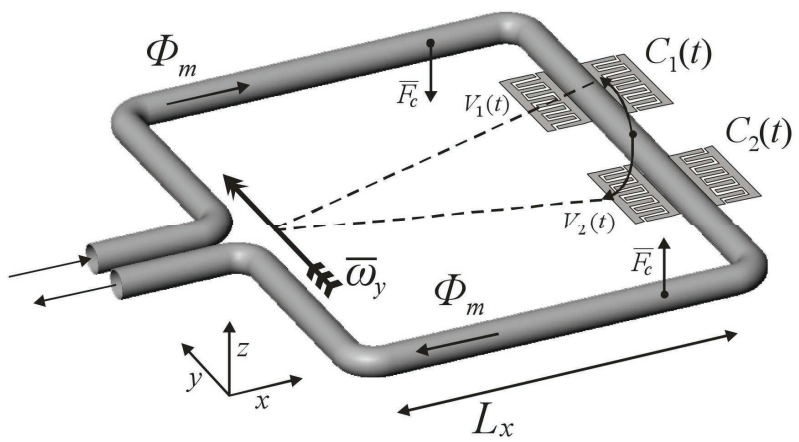

(b)

Figure 1: Operating principle of the Coriolis mass flow sensor. Actuation of the tube in torsion mode $\omega_{x}$ (a) results in Coriolis forces $F_{C}$ and a flapping motion proportional to the mass flow $\Phi_{m}$ inside the tube. Actuation of the flapping mode $\omega_{y}$ (b) results in Coriolis forces that induce a torsional motion. 


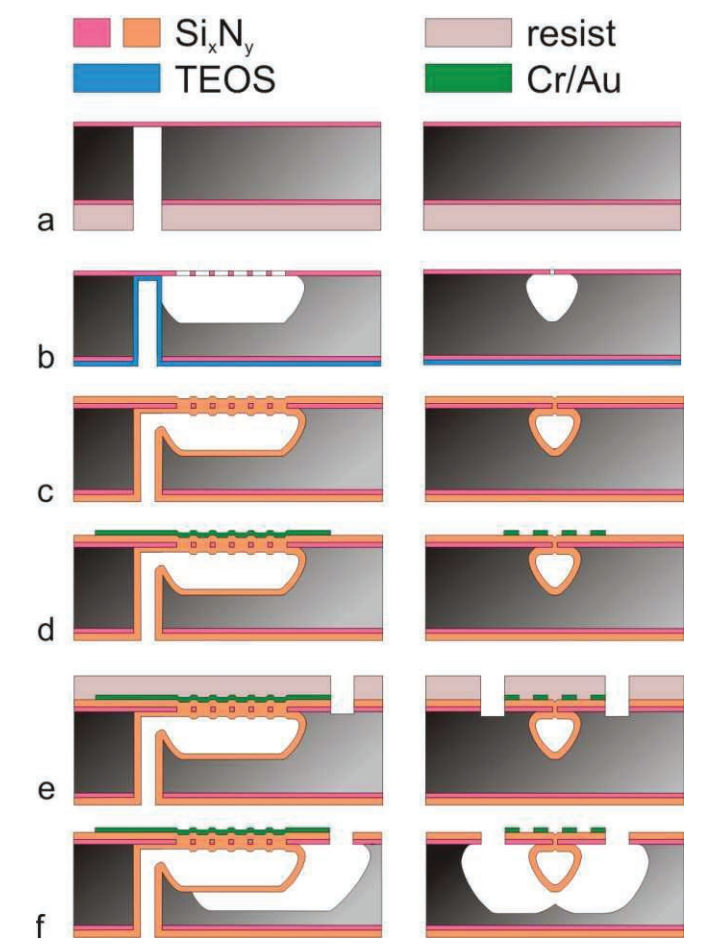

Figure 2: Outline of the fabrication process. Left column: cross-section along the length of the tube. Right column: cross-section perpendicular to the sensor tube.

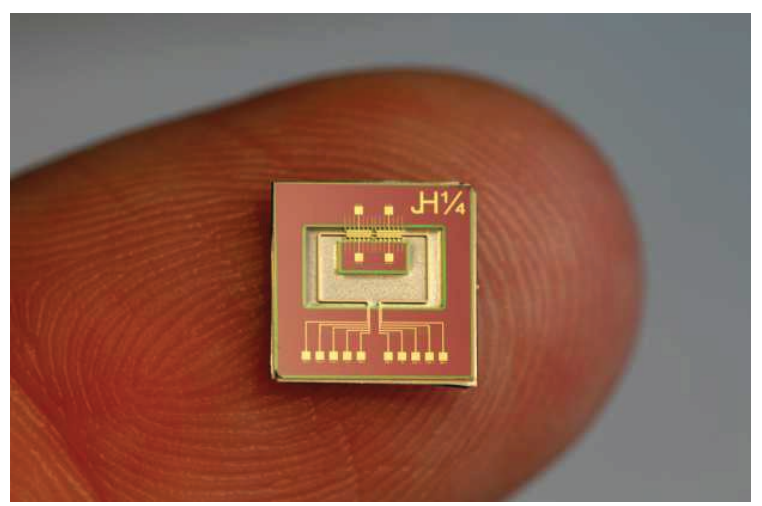

Figure 3: Photograph of the sensor chip, which measures $7.5 \mathrm{~mm} \times 7.5 \mathrm{~mm}$. Bond-pads for electrical connections are at the front side of the chip. Fluidic connections are located at the back.

\section{ELECTROSTATIC ACTUATION}

As indicated in figure 1, comb-shaped electrodes are used for both the actuation and detection capacitors. This prevents squeezed film damping and also simplifies the fabrication process significantly since both electrodes of the capacitors can be deposited in a single step. The electrodes can be used for actuation because the stress induced by the deposition of the metal layer results in a slight out-of-plane bending of the tube, as can be seen in the SEM photographs shown in figure 4. 


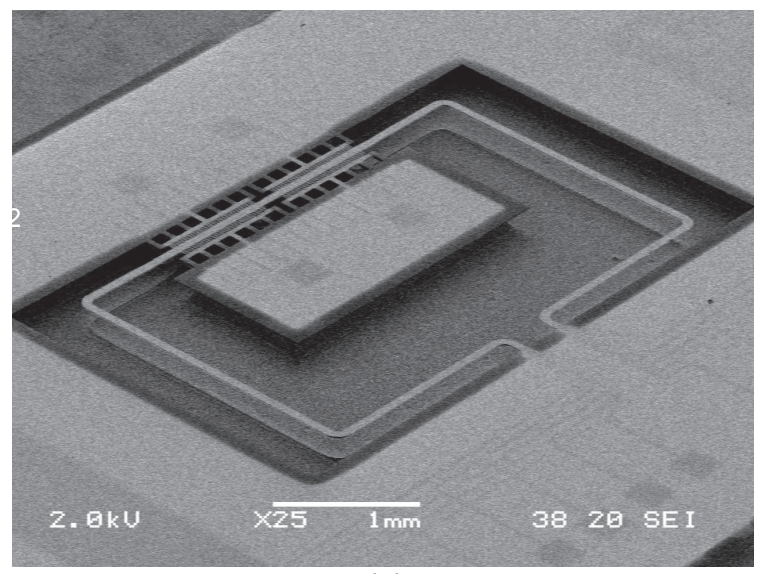

(a)

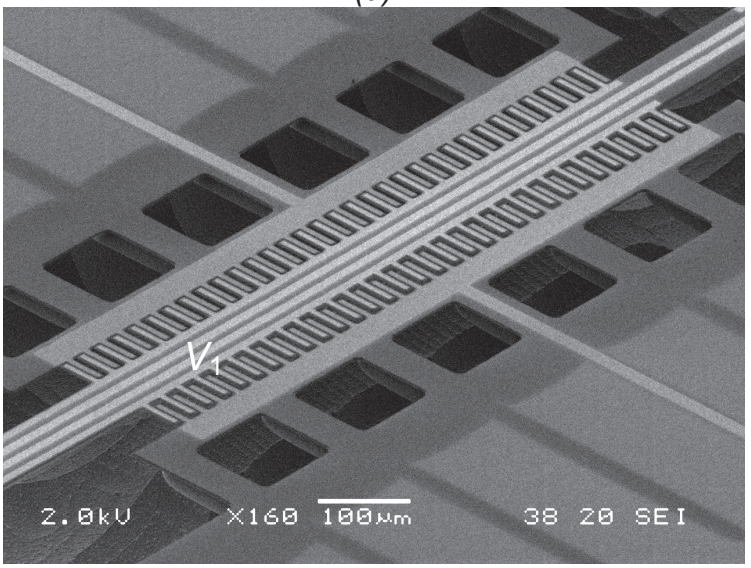

(b)

Figure 4: SEM photograph showing the rectangular sensor tube and the electrodes for actuation and readout (a), and detailed view of one of the actuation and readout capacitors (b). The stress induced by deposition of the metal layer results in a slight out-of-plane static deflection of the tube, so that the comb fingers attached to the tube are elevated by about $2 \mu \mathrm{m}$ with respect to the fixed comb fingers.

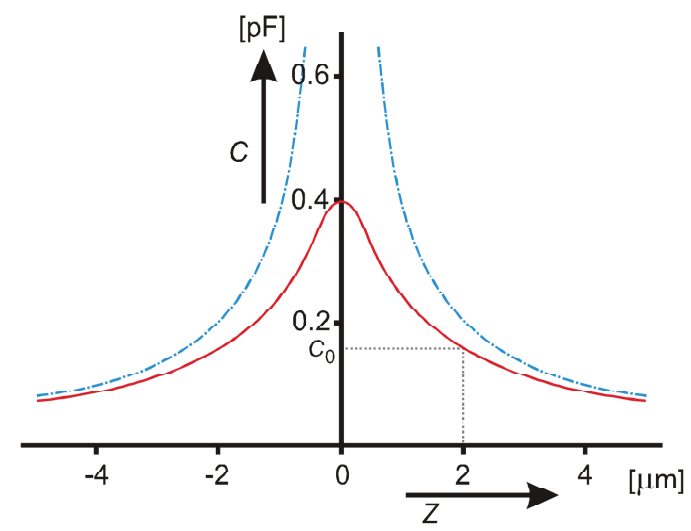

Figure 5: Calculated capacitance (solid line) between two comb-shaped electrodes as a function of the position of the tube with respect to the silicon surface. The initial position is approximately $2 \mu \mathrm{m}$. The dashed lines indicate the capacitance of a corresponding parallel plate capacitance.

Figure 5 shows the calculated capacitance between the comb-shaped electrodes. The maximum in capacitance occurs when the electrodes are exactly inline. In rest, the sensor tube is displaced by approximately $2 \mu \mathrm{m}$. A voltage applied between the electrodes results in an attractive electrostatic force, which is proportional to the voltage squared and to the derivative of the capacitance with respect to position. In order to actuate the flapping mode $\omega_{y}$ both actuation capacitors are driven with the same voltage, i.e.: 


$$
V_{1}=V_{2}=V_{\text {bias }}+V_{a} \cdot \sin (\omega t)
$$

Usually $V_{\text {bias }}$ is taken equal to $V_{a}$ so that the structure is actuated at frequency $\omega$. Alternatively, using zero bias voltage results in actuation at twice the frequency, which can be useful to prevent electrical crosstalk.

For actuation of the torsional mode $\omega_{x}$ the two actuation voltage need to be 90 degrees out of phase:

$$
\begin{aligned}
& V_{1}(t)=V_{a} \cdot \sin (\omega t) \\
& V_{2}(t)=V_{a} \cdot \sin \left(\omega t+\frac{\pi}{2}\right)
\end{aligned}
$$

Due to the quadratic nature of the electrostatic forces the result is that the structure is excited at twice the frequency with 180 degrees phase difference:

$$
\begin{aligned}
& F_{1}(t) \propto V_{1}^{2}=\frac{1}{2} V_{a}^{2} \cdot(1-\cos (2 \omega t)) \\
& F_{2}(t) \propto V_{2}^{2}=\frac{1}{2} V_{a}^{2} \cdot(1-\cos (2 \omega t+\pi))
\end{aligned}
$$

For both types of actuation the displacement amplitude of the tube is limited by the initial electrode distance of $2 \mu \mathrm{m}$, which is reached at actuation voltage amplitudes $V_{a}$ in the order of $10 \mathrm{~V}$. A further increase in voltage does not increase the displacement amplitude. We are currently investigating the use of different, e.g. pulsed, actuation voltages to increase the displacement amplitude, since this will directly result in an improved signal to noise ratio.

\section{MEASUREMENT RESULTS}

Figure 6 shows a schematic drawing of the measurement setup used for flow measurements. A syringe pump was used to generate volume flows between 0 and $1.2 \mathrm{~cm}^{3} / \mathrm{hr}$ through the chip. During the measurement the sensor output signal is recorded, together with the inlet pressure of the liquid using a separate pressure sensor, which is used as a reference for the volume flow rate. The pressure sensor is calibrated for volume flow at the highest flow level generated by the syringe pump of $1.2 \mathrm{~cm} / \mathrm{hr}$, assuming a linear dependence between volume flow and pressure.

The output of the sensor is obtained by measuring the capacitances $C_{1}$ and $C_{2}$, which are located opposite to the actuation capacitors (see Fig. 4a). The phase difference between these signals is proportional to the mass flow, as explained in [11].

Figure 7 shows a flow measurement using flapping actuation. In this case the torsional mode is excited by the Coriolis force. Figure 8 shows a flow measurement using torsion actuation. In this case the flapping mode is excited by the Coriolis force. The measurements clearly show that the structure is more sensitive with torsion actuation, resulting in a larger change in phase and better signal-to-noise ratio. This is in agreement with the theoretical model of the sensor structure since the flapping mode is more efficiently excited by the Coriolis force. Furthermore, the readout electrodes are located relatively close to the axis of the torsion mode so that a flapping motion gives a much larger signal than a torsional motion. Even with torsion actuation the signal-to-noise ratio is worse than previously found with Lorentz for actuation [11]. This is a direct consequence of the reduced actuation amplitude.

\section{CONCLUSIONS}

We have designed and realized a micro Coriolis mass flow sensor for a flow range of $0-1.2 \mathrm{~g} / \mathrm{hr}$ with electrostatic actuation and capacitive readout. Comb-shaped capacitor electrodes with a static displacement of approximately $2 \mu \mathrm{m}$ were used. These structures have the advantage that only a single metal layer is needed during fabrication and excessive squeezed film damping which would occur in a traditional parallel-plate configuration is avoided. As a result, the sensor can operate in air at atmospheric pressure with a quality factor of approximately 40 , thus eliminating the need for vacuum operation and integrated getter materials.

\section{ACKNOWLEDGEMENTS}

This research was financed by the Dutch MicroNed, PIDON High Tech Factory and Kenniswerkers programs. The authors would like to thank the industrial partners in these projects for many fruitful discussions. 


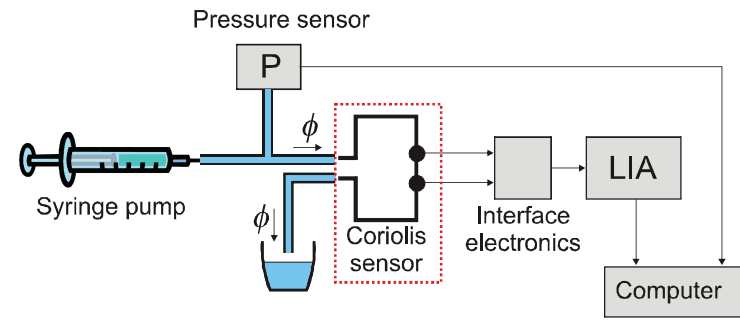

Figure 6: Overview of the flow measurement setup. Lock-in amplifiers (LIA) are used to detect the sensor output signals and calculate the phase difference.

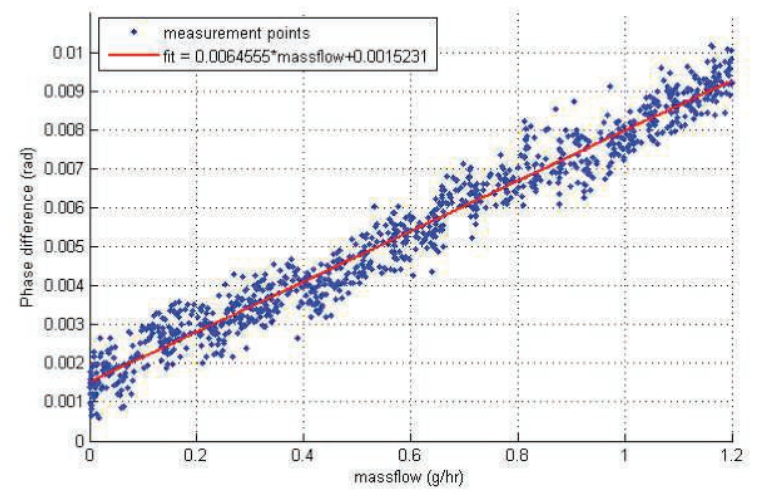

Figure 7: Mass flow measurement using flapping mode $\left(\omega_{y}\right)$ actuation. In this case the Coriolis force excites the torsion mode.

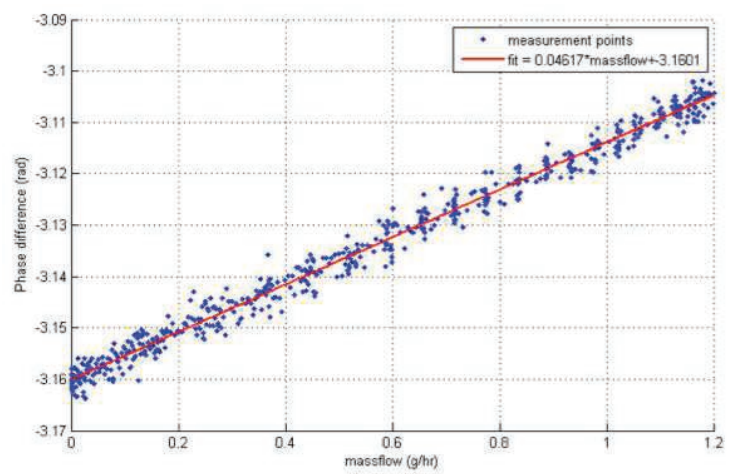

Figure 8: Mass flow measurement using torsion mode $\left(\omega_{x}\right)$ actuation. In this case the Coriolis force excites the flapping mode.

\section{REFERENCES}

[1] Y. Mizuno, M. Liger and Y.-C. Tai, Proc. MEMS 2004, pp. 322-325.

[2] M. Dijkstra, M.J. de Boer, J.W. Berenschot, T.S.J. Lammerink, R.J. Wiegerink and M. Elwenspoek, Proc. MEMS 2007, pp. 123-126.

[3] M. Dijkstra, T.S.J. Lammerink, M.J. de Boer, R.J. Wiegerink and M. Elwenspoek, Proc. MEMS 2009, pp. 479-482.

[4] R.C. Baker, Flow. Meas. Instrum., 5, 1994, pp. 229-246.

[5] M. Anklin, A. Drahm Wand Rieder, Flow. Meas. Instrum., 17, 2006, pp. 317-323.

[6] A. Mehendale and P.P.L. Regtien, 3rd Int. Symp. Sens. Sci. (Jülich, Germany, Jul. 18-21), 2005

[7] J. Haneveld, T.S.J. Lammerink, M. Dijkstra, H. Droogendijk, M.J. de Boer and R.J. Wiegerink, Proc. MEMS 2008, pp. 920-923.

[8] P. Enoksson, G. Stemme and E. Stemme, J. MEMS, 6 (1997), pp. 119-125.

[9] D. Sparks, R. Smith, J. Cripe, R. Schneider, N. Najafi, Proc. IEEE Sensors Conference, 2003, pp. 9092.

[10] J. Haneveld, T.S.J. Lammerink, M.J. de Boer and R.J. Wiegerink, Proc. MEMS 2009, pp. 463-466.

[11] J. Haneveld, T.S.J. Lammerink, M.J. de Boer, R.G.P. Sanders, A. Mehendale, J.C. Lötters, M. Dijkstra and R.J. Wiegerink, J. Micromech. Microeng., 20, 2010, 125001. 